\title{
Über eine langperiodische Struktur der Cellulosemoleküle und der aus Cellulose aufgebauten Pflanzenfasern
}

\begin{abstract}
Von G. V. Schulz und E. HusemanN
Aus dem Institut für physikalische Chemie der Universität Rostock und der Forschungsabteilung für makromolekulare Chemie des chemischen Laboratoriums der Universität Freiburg i. Br.

(Z. Naturforschg. 1, 268-280 [1946]; eingegangen am 28. März 1946)

Cellulose aus Pflanzenfasern (Baumwolle, Ramie) besteht aus linearen Kettenmolekülen, welche etwa 3200 in $\beta$-glucosidischer Bindung verknüpfte Glucosereste enthalten. Fraktionierversuche an nativer Cellulose zeigen, daß deren Moleküle (ganz oder annähernd) gleiche Länge besitzen. Die Molekülgrößen in abgebauten Cellulosen sind séhr viel einheitlicher als zu erwarten wäre, wenn alle Bindungen in der Kette gleichartig wären. Eine genaue Untersuchung der Molekülgrößenverteilung ergibt, daß in regelmäßigen Abständen von etwa 500 Glucoseresten leichter aufspaltbare Bindungen in der Kette vorhanden sind. Zum gleichen Ergebnis führt eine reaktionskinetische Analyse der Abbauvorgänge. — Ǔbermikroskopische Aufnahmen von nach einer neuen Methode präparierten Naturfasern zeigen, daß sich die Periodizität der Moleküle in eine Periodizität des Faseraufbaus fortsetzt. Künstliche Fasern besitzen diese Periodizität nicht. - Aus dem Befund ergeben sich Konsequenzen über die Entstehung der makromolekularen Stoffe im Organismus.
\end{abstract}

$\mathrm{W}$ ährend der tierische Organismus seinen Körper aus Bauelementen zusammensetzt, deren Grundsubstanzen die Faserproteine sind (Kollagen, Myosin usw.), erhält der pflanzliche Organismus die Festigkeit seiner Gestalt vorwiegend durch Polysaccharide, deren Hauptvertreter die Cellulose ist. Nun ergaben in den letzten Jahren die Arbeiten einer Reihe von Forschern, daß die Moleküle der Eiweißstoffe nach periodischen Gesetzmäßigkeiten aufgebaut sind. Der chemischen Analyse zufolge bestehen ganzzahlige stöchiometrische Verhältnisse zwischen den einzelnen, ein bestimmtes Proteinmolekül aufbauenden Aminosäuren ${ }^{1}$. Andrerseits haben röntgenographische Untersuchungen amerikanischer ${ }^{2}$, englischer ${ }^{3}$ und deutscher $^{4}$ Forscher ergeben, daß Faserproteine zahlreiche Interferenzen zeigen, aus denen Gitterebenen mit sehr großen Abständen (bis zu $642 \AA)^{4}$ errechnet werden können. Schließlich ist es gelungen, diese morphologischen Befunde mit den Ergebnissen der chemischen Analyse derart in Übereinstimmung $\mathrm{zu}$ bringen, daß man den aus den Interferenzen errechneten Abständen mit hoher Wahrscheinlichkeit bestimmte Aminosäuren zuordnen konnte ${ }^{4}$.

1 M. Bergmann und C. Niemann, J. biol. Chem. 115, 77 [1936]; u. a. Publikationen.

${ }^{2}$ R. W. G. W y ck of $\mathrm{f}$ und R. B. Corey, Science [New York] 81, 749 [1934]; J. biol. Chem. 114, 407 [1936]; u. a. Publikationen.
Demgegenüber scheinen die Polysaccharide einen sehr viel eintönigeren Aufbau zu besitzen. Beispielsweise ist der einzige bisher nachgewiesene Baustein der Cellulose die Glucose; und speziell die Cellulose der eigentlichen Faserstoffe (Baumwolle, Ramie u. a.) besteht aus langen Kettenmolekülen, in welchen etwa 3000 Glucosereste in linearer Ạnordnung $\beta$-glucosidisch miteinander verknüpft sind.

Wir fanden in einer Reihe von Untersuchungen, daß auch in den Cellulosemolekülen Perioden von allerdings sehr großer Länge vorkommen. Das ergibt sich einmal daraus, daß das Cellulosemolekül, wenn man es einem hydrolytischen oder oxydativen Abbau unterwirft und diesen in bestimmten Stadien unterbricht, in Bruchstücke von gleicher Länge zerfällt. Dieses könnte nicht der Fall sein, wenn alle Glucosereste in der Kette gleichartig miteinander verknüpft wären; denn dann müßte der Abbau ein Gemisch verschiedener Bruchstücke ergeben, deren Kettenlänge über einen sehr großen Bereich statistisch schwankt. Aus der Länge der aufgefundenen Bruchstücke ergibt sich, daß in regelmäßigen Abständen von ungefähr 500 Glucoseresten rascher spaltbare Bindungen in das Cellulosemolekül eingebaut sind (Abschnitt I).

3 J. D. Bernal u. D. Crow foot, Nature [London] 133, 749 [1934]; Cr ow fo ot, ebenda 135, 591 [1935].

${ }_{4}^{4}$ O. Kratky u. A. Sekora, J. makromol. Chem. 1, 113 [1943]. 
Die leichter angreifbaren Bindungen machen sich ferner bemerkbar, wenn man den Abbau reaktionskinetisch verfolgt. Dieser geht àm Anfang der Reaktion sehr viel rascher vor sich als in den späteren Stadien, und aus einer quantitativen Untersuchung dieser Verhältnisse lassen sich die reaktionskinetischen Konstanten der verschiedenen Bindungen ermitteln. Auf Grund der so erhaltenen Zahlenwerte kann man dann versuchen, ein chemisches Modell für die Sondergruppen aufzustellen (Abschnitt II).

Die periodische Struktur der Einzelmoleküle setzt sich in übermolekulare Perioden fort, die beim Aufbau der Fasern eine Rolle spielen. Für eine röntgenographische Analyse sind diese Perioden allerdings bei den heutigen experimentellen Hilfsmitteln $\mathrm{zu}$ groß. Jedoch ist es gelungen, sie mit dem Ubermikroskop sichtbar zu machen, wobei sich eine zahlenmäßige UUbereinstimmung des morphologischen und des chemischen Befundes ergab (Abschnitt III).

Im Abschnitt IV versuchen wir, aus unseren Ergebnissen einige Schlußfolgerungen über die Entstehung der makromolekularen Stoffe im Organismus im Zusammenhang mit dessen submikroskopischer Struktur zu ziehen.

Wir geben in diesem Bericht einen Uberblick über eine Reihe von Arbeiten, die wir mit mehreren Mitarbeitern in den letzten 6 Jahren über dieses Gebiet ausgeführt haben. Wegen der äußeren Umstände konnte bisher nur ein Teil von ihnen publiziert werden.

\section{Nachweis der Perioden}

\section{a) Charakterisierung des Ausgangsmaterials}

Als Ausgangsmaterial dienten uns Baumwolle und Ramie. Die Baumwolle war vollständig unbehandelt, die Ramie leider schon einem leichten Bleichprozeß unterworfen. Wir orientierten uns zunächst über den molekularen Aufbau der Ausgangsprodukte, indem wir deren mittleren Polymerisationsgrad bestimmten und dann durch Zerlegung in Fraktionen durch fraktionierte Fällung prüften, wieweit die Stoffe molekular einheitlich oder uneinheitlich waren. Die Molekulargewichtsbestimmungen geschahen auf viscosimetrischem Wege, nachdem wir vorher durch größere Versuchsreihen die Beziehung zwischen Viscositätszahl und osmotisch bestimmtem Molekulargewicht ermittelt hatten ${ }^{5}$.

\begin{tabular}{|c|c|c|c|c|c|}
\hline Fraktion & $\%$ & $\bar{P}$ & Fraktion & ${ }^{0} / 0$ & $\bar{P}$ \\
\hline \multicolumn{6}{|c|}{ 1. Baumolle 6} \\
\hline $\begin{array}{l}1 \\
2 \\
3\end{array}$ & $\begin{array}{r}1,0 \\
17,0 \\
30,0\end{array}$ & $\begin{array}{r}470 \\
1200 \\
2940\end{array}$ & $\begin{array}{l}4 \\
5 \\
-\end{array}$ & $\begin{array}{r}35,9 \\
14,5 \\
-\end{array}$ & $\begin{array}{c}3140 \\
3200 \\
-\end{array}$ \\
\hline \multicolumn{6}{|c|}{ 2. $\mathrm{Ramie}^{7}$} \\
\hline $\begin{array}{l}1 \\
2 \\
3 \\
4 \\
5\end{array}$ & $\begin{array}{r}3,20 \\
10,64 \\
5,32 \\
14,36 \\
9,57\end{array}$ & $\begin{array}{c}\text { picht meßbar } \\
600 \\
926 \\
1140 \\
1550\end{array}$ & $\begin{array}{r}6 \\
7 \\
8 \\
9 \\
10\end{array}$ & $\begin{array}{r}15,95 \\
9,57 \\
12,24 \\
11,70 \\
7,45\end{array}$ & $\begin{array}{l}1790 \\
2220 \\
2530 \\
3100 \\
3180\end{array}$ \\
\hline
\end{tabular}

Tab. 1. Fraktionierung von Fasercellulosen.

Der mittlere Polymerisationsgrad $\overline{\mathrm{P}}$ der Baumwoll-Cellulose war 2600, der Ramie-Cellulose 2000. Zerlegt man die beiden Cellulosen durch fraktionierte Fällung ${ }^{6}$, so erhält man das in Tab. 1 dargestellte Resultat. Man sieht, daß die Baumwoll-Cellulose zu etwa $80 \%$ aus Molekülen mit einem Polymerisationsgrad über 3000 , die Ramie-Cellulose zu nur etwa $25 \%$ aus so großen Molekülen besteht. Es ist sehr wahrscheinlich, daß die nativen Moleküle gleichartige Ketten mit einem Polymerisationsgrad von etwa 3200 sind, und daß durch die nachfolgende Behandlung ein Teil der Moleküle chemisch abgebaut worden ist. Für die Beurteilung der hier geschilderten Versuchsergebnisse ist es wichtig, daß in beiden Ausgangsprodukten der Polymerisationsgrad der überwiegenden Hauptmenge weit über 1000 liegt.

Später fand Gralén ${ }^{8}$ im Svedbergschen Institut mit der Ultrazentrifuge sehr viel höhere Teilchengewichte für Cellulosen. Die Diskrepanz mit unseren Ergebnissen dürfte in folgendem liegen: Bei unseren Versuchen wurde durch vorherige alkalische Behandlung (unter Stickstoff) alles Pektin und alle ligninhaltigen Bestandteile aus den Fasern ausgewaschen (ebenso die Fette und Wachse), während Gralén keine Reinigung vor der Auflösung vornahm. Nun ist es sehr wahrscheinlich, daß das Lignin und andere „Kittsubstanzen" im Holz - und damit wohl auch in der Baumwolle - mit der Cellulose chemisch verbunden sind. Durch eine derartige „Kittsubstanz" könnten in der Baumwolle Elementarketten der Cellulose vom Polymerisationsgrad $3200 \mathrm{zu}$ höhermolekularen Aggregaten verknüpft sein, welche bei der von uns angewandten Behandlung mit Alkali auseinanderfallen. Dafür spricht auch, daß die Gralénsche Cellulose im Gegensatz zu unserer außerordentlich uneinheitlich ist.

5 E. Husemann u. G. V.Schulz, Z. physik. Chem. (B) $52,1[1942]$.

${ }_{6}^{6}$ G. V.Schulz u. E. Husemann, ebenda S. 23.

7 E. Hus em ann, unveröffentlicht.

8 Nils Gralén, Dissertat. Uppsala 1944. 
Der Nachweis für die hauptvalenzmäßige Verknüpfung der Glucosereste im Cellulosemolekül war bekanntlich früher von Staudinger und Daumiller ${ }^{9}$ erbracht worden.

\section{b) Die Verteilung der Kettenlängen in abgebauten Cellulosen}

Unterwirft man die Cellulose einem hydrolytischen oder oxydativen Abbau, dann sind zwei Dinge zu erwarten:

1. daß die mittlere Kettenlänge abnimmt;

2. daß die Uneinheitlichkeit des Materials immer mehr zunimmt, falls nämlich alle Bindungen in der Kette die gleiche Spaltungswahrscheinlichkeit haben.

Wir untersuchten dieses, indem wir den Abbau nach verschiedenen Zeiten unterbrachen und die mehr oder weniger stark abgebaute Cellulose quantitativ in Fraktionen zerlegten ${ }^{\mathbf{1 0}} \mathbf{1 1}$.

\begin{tabular}{|c|c|c|c|c|c|}
\hline \multicolumn{2}{|c|}{$\begin{array}{c}\bar{P}=960 \\
\text { Pıäparat } 3\end{array}$} & \multicolumn{2}{|c|}{$\begin{array}{c}\bar{P}=425 \\
\text { Präparat } 6\end{array}$} & \multicolumn{2}{|c|}{$\begin{array}{c}\bar{P}=147 \\
\text { Präparat } 15\end{array}$} \\
\hline$\%$ & $\bar{P}$ & $\%$ & $\bar{P}$ & $\%$ & $\bar{P}$ \\
\hline 6.7 & 136 & 1,0 & 25 & 7,0 & 40 \\
\hline 6,4 & 518 & 4,5 & 99 & 8,6 & 107 \\
\hline 8,0 & 835 & 4,3 & 185 & 14,4 & 155 \\
\hline 14,4 & 990 & 17,3 & 290 & 28,1 & 220 \\
\hline 9,0 & 1360 & 13,3 & 400 & 7,7 & 276 \\
\hline 8,4 & 1510 & 9,2 & 470 & 17,3 & 328 \\
\hline 17,1 & 1720 & 15,1 & 490 & 7,7 & 450 \\
\hline 14,3 & 2100 & 14,6 & 580 & 9,2 & 560 \\
\hline 10,5 & 2490 & 4,8 & 760 & & \\
\hline 5,2 & 2900 & 15,9 & 900 & & \\
\hline
\end{tabular}

Tab. 2. Fraktionierung von 3 Abbaustadien von Cellulose aus Ramiefasern (Hydrolyse mit $\mathrm{KHSO}_{4}$ ).

Tab. 2 zeigt solche Untersuchungen an 3 Abbaustadien von Ramie aus einer größeren Untersuchungsreihe des einen von $u_{n} \mathrm{~s}^{12}$. Man sieht schon aus dieser Fraktioniertabelle, daß die Uneinheitlichkeit zunächst zunimmt (Präp.3), dann. aber wieder stark abnimmt, denn das Präp. 6 zeigt eine deutliche Anreicherung der Polymerisationsgrade in der Umgebung von 500. Bei weiterem Abbau

- H. Staudinger u. G. Daumiller, Lieb. Ann. Chem. 529, 219 [1937].

10 Uber die Methode vergl. G. V. Schulz u. E. Hus ema $n$, Zitat 6 .

11 Da Cellulose selbst sehr schwer fraktionierbar ist, wurde das Material unter Aufrechterhaltung des Polymerisationsgrades (polymeranalog) nitriert, und dann die weiteren Versuche an den Cellulosenitraten unternommen. Über polymeranaloge Nitrierung vergl. $H$. Stauding er u. R. Mohr, Ber. dtsch. chem. Ges. 70, 2269 [1937].
(Präp. 15) nimmt die Uneinheitlichkeit wieder zu.

Noch deutlicher erkennt man das, wenn man aus den Fraktioniertabellen die Verteilungskurven der untersuchten Stoffe ausrechnet. Die Verteilungskurve stellt graphisch dar, wie groß der Gewichtsanteil $m_{P}$ jedes einzelnen Polymerisationsgrades $P$ im Gemisch ist. Sie kann nach einem von dem einen von uns angegebenen Verfahren aus der Fraktio-
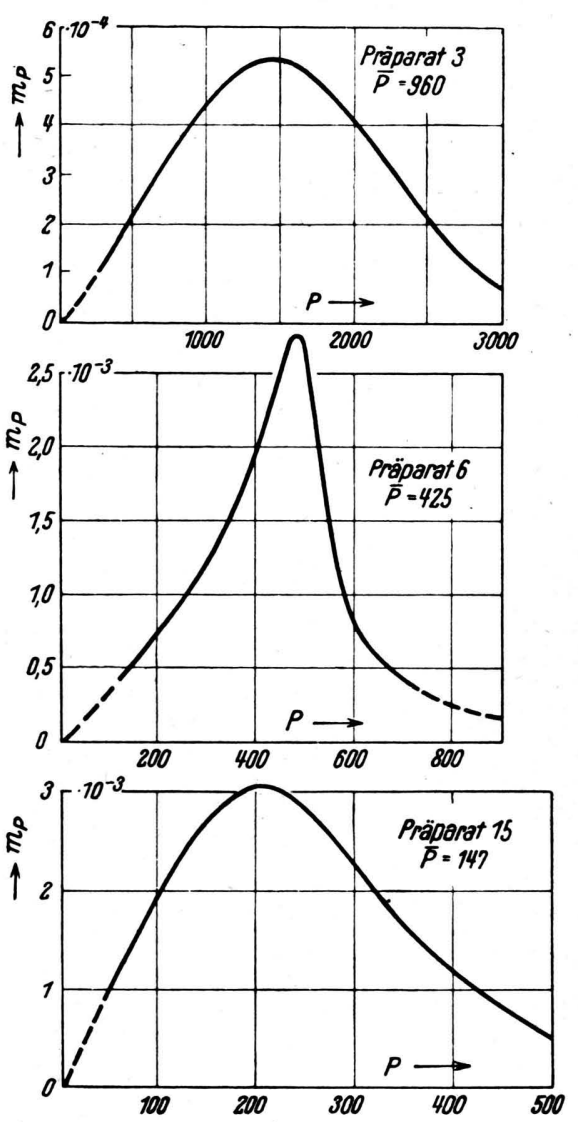

Abb. 1. Verteilungskurven verschieden stark abgebauter Ramie-Cellulose.

niertabelle berechnet werden ${ }^{13}$. In Abb. 1 sind die so ermittelten Verteilungskurven der drei Präparate aus Tab. 2 dargestellt. Man sieht jetzt unmittelbar, wie im Präp. 6 der Polymerisationsgrad 500 außerordentlich stark angereichert ist. Das weiter abgebaute Präp. 15 ist wieder sehr viel uneinheitlicher.

Wären alle Bindungen im Cellulosemolekül gleich, so müßte in den Verteilungskurven ein gleichmäßiger UUbergang vom Präp. 3 zum Präp. 15

12 E. Husemann, unveröffentlichte Versuche.

13 G. V.Schulz, Z. physik. Chem. (B) 47, 155 [1940]. 
auftreten. Die sehr viel größere Einheitlichkeit des Präp. 6 mit dem ausgeprägten Maximum beim Polymerisationsgrad 500 zeigt, daß in regelmäßigen Abständen von etwa 500 Glucoseresten schneller spaltende Gruppen in die Cellulosekette eingebaut sind.

\section{c) Der Gang der Uneinheitlichkeit bei zunehmen- dem Abbau}

Noch besser und etwas weniger umständlich als mit der Fraktioniermethode kann man die Uneinheitlichkeit bei fortschreitendem Abbau nach einer Methode verfolgen, die eine Maßzahl für die Uneinheitlichkeit der Präparate direkt zu bestimmen erlaubt. Diese Methode beruht auf folgender Uberlegung:

Die Cellulosen und ihre Derivate gehorchen sehr genau dem Staudingerschen Viscositätsgesetz $^{5,14}$

$$
Z_{\eta}=K_{m} \cdot P \text {. }
$$

Hierbei ist $P$ der Polymerisationsgrad, $Z_{\eta}$ die Viscositätszahl und $K_{m}$ eine experimentelle Konstante. Die Viscositätszahl ist definitionsgemäß

$$
Z_{\eta} \equiv\left[\frac{\eta_{s p}}{c}\right]_{c=0}
$$

worin $\eta_{s p}$ die spezifische Viscosität* einer Lösung der Konzentration $c$ (in $g / l$ ) bedeutet. Gleichung (1) gilt jedoch nur, wenn die Substanz einheitlich ist. Das liegt daran, daß bei der osmotischen Messung ein anderer Durchschnittswert $\bar{P}$ für den Polymerisationsgrad herauskommt als bei der viscosimetrischen Messung $\left(P_{\eta}\right)^{15}$. Wie W. $\mathrm{Ker} \mathrm{n}^{16}$ nachwies, ist bei einheitlichen Stoffen $P_{\eta} / \bar{P}=1$, dagegen bei uneinheitlichen Stoffen $P_{\eta} / \bar{P}>1$. Als Maß der Uneinheitlichkeit können wir daher den Ausdruck

14 G. V. Schul z, J. makromol. Chem. 1, 146 [1943].

* Die spezifische Viscosität ist

$$
\eta_{s p}=\frac{\eta \text { (Lösung) }-\eta \text { (Lösungsmittel) }}{\eta \text { (Lösungsmittel) }} .
$$

15 Wie an anderer Stelle ausgeführt worden ist, ist $\bar{P}=1 / \underset{P=1}{P} \bar{\Sigma}_{P}^{\infty}\left(m_{P} / P\right)$, dagegen $\quad P_{\eta} \underset{P=1}{P}=\sum_{P}^{\infty} m_{P}$, wobei $m_{P}$ die Masse (in g) des Polymeren vom Polymerisations$\operatorname{grad} P$ in $1 \mathrm{~g}$ des Ausgangsgemisches ist. Bei Kenntnis der Verteilungsfunktion kann man $P_{\eta}$ und $\bar{P}$ berechnen, indem man die Summation ausführt. G. V. Schulz, Z. physik. Chem. (B) 32, 27 [1936]; 47, 155 [1940]; sowie Zitat ${ }^{14}$.

$$
U=\frac{P_{\eta}}{\bar{P}}-1
$$

verwenden, der bei einheitlichen Stoffen gleich 0 ist, sonst aber endliche Werte (im allgemeinen zwischen 0 und 1,5) annimmt.

Zur Bestimmung von $U$ haben wir also eine osmotische und eine viscosimetrische Messung miteinander zu vergleichen, was folgendermaßen geschieht. Aus dem viscosimetrisch bestimmten $Z_{\eta}$ und dem osmotisch bestimmten $\bar{P}$ rechnet man nach (1) einen $K_{m}$-Wert aus. Wegen der verschiedenen Mittelung der beiden Meßmethoden ist dieser $K_{m}$-Wert umso höher, je uneinheitlicher der Stoff ist. Bezeichnen wir den an einem beliebigen Stoff bestimmten Wert als $K_{m}$, dagegen den einer einheitlichen Substanz als $k_{m}$, so gilt offenbar $K_{m} / k_{m}=P_{\eta} / \bar{P}$, was in (3a) eingesetzt

$$
U=\frac{K_{m}}{k_{m}}-1
$$

ergibt. Die für jede polymerhomologe Reihe charakteristische $k_{m}$-Konstante bestimmt man an sorgfältig hergestellten Fraktionen unter Verwendung eines Korrekturfaktors von $4 \%^{15}$.

Wir wollen uns nun zunächst darüber orientieren, welchen Gang von $U$ man mit steigendem Abbaugrad unter verschiedenen Voraussetzungen zu erwarten hat. Hierzu hat man zunächst die Verteilungsfunktion (d.h. das zu jedem $P$ gehörige $m_{P}$ ) unter definierten Bedingungen auszurechnen. Ist diese bekannt, so kann man die obigen Summenausdrücke bilden, aus welchen sich bestimmte Werte für $U$ ergeben. Geht man von einem molekular einheitlichen Stoff aus, so wächst $U$ mit steigendem Abbaugrad $\beta$ vom Anfangswert 0 auf einen bestimmten endlichen Wert an. Als Abbaugrad $\beta$ bezeichnen wir den Quotienten aus dem ursprünglichen Polymerisationsgrad $P_{0}$ und dem des abgebauten Produktes $\bar{P}$

$$
\beta=P_{o} / \bar{P} .
$$

Sind alle Bindungen im Kettenmolekül gleich, so steigt $U$, wie man berechnen $\mathrm{kann}^{17}$, entsprechend der Kurve J in Abb. 2 vom Wert 0 bis auf den Wert 1 an. Sind schneller spaltende Bindungen in regelmäßigen Abständen in das Molekül eingebaut, so geht die $U$-Kurve durch ein Minimum, um sich erst bei sehr hohen Abbaugraden wieder dem

16 Ber. dtsch. chem. Ges. 68, 1439 [1935].

${ }^{17}$ G. V. Schul z, Z. physik. Chem. (B) 51, 127 [1942]. 
Wert 1 zu nähern. Das Minimum in dieser-Kurve ist (wie schon qualitativ verständlich) um so tiefer, je größer der Unterschied der Geschwindigkeitskonstanten $k_{a}$ und $k_{b}$ der beiden Sorten von Bindungen ist. In Abb. 2 sind die Kurven für verschiedene

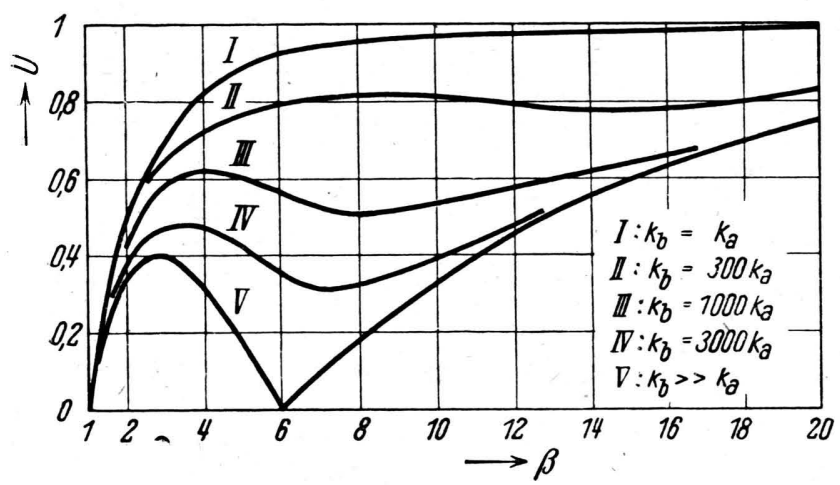

Abb. 2. Uneinheitliehkeit $U$ in Abhängigkeit vom Abbaugrad $\beta$ bei verschiedenen Verhältnissen der beiden Geschwindigkeitskonstanten $k_{a}$ und $k_{b}$ (berechnet).

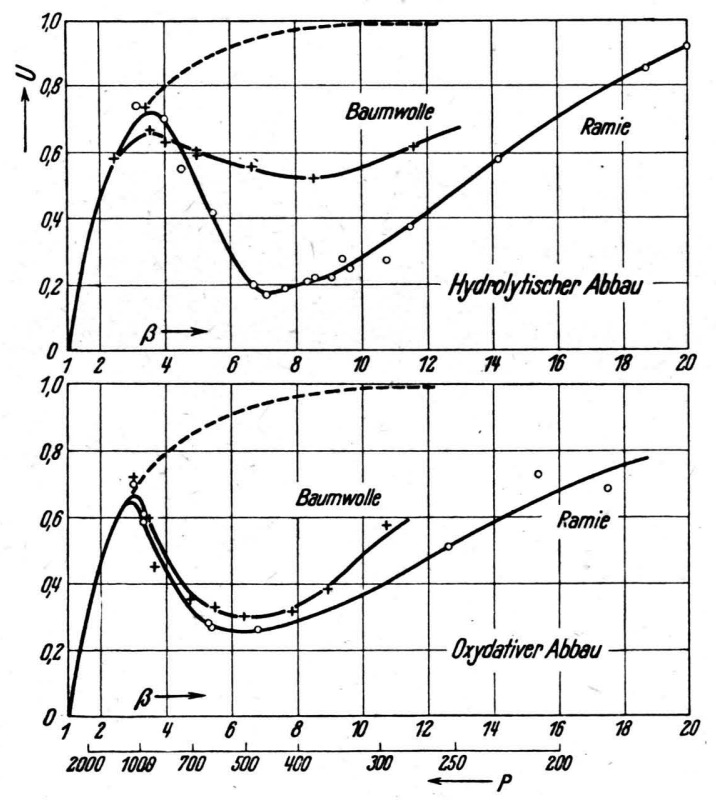

Abb. 3. Uneinheitlichkeit in Abhängigkeit vom Abbaugrad, (experimentell).

Werte des Verhältnisses der beiden Geschwindigkeitskonstanten berechnet, und zwar unter der Annahme, daß $P_{0}=3000$ ist und der Abstand der schneller spaltenden Gruppen (mit der Geschwindigkeitskonstante $k_{b}$ ) 500 Glucosereste beträgt ${ }^{18}$.

In dieser Weise wurde die Uneinheitlichkeit bei wachsendem Abbaugrad an der in Tab. 1 bereits ${ }^{18}$ G. V. S c h u l z, ebenda 52, 50 [1942].

\begin{tabular}{|c|l|l|l|c|c|}
\hline $\begin{array}{c}\text { Nr. des } \\
\text { Präpa- } \\
\text { rates }\end{array}$ & $\begin{array}{c}\bar{P} \\
\text { (osmot.) }\end{array}$ & $\beta$ & \multicolumn{1}{|c|}{$Z_{\eta}$} & $\begin{array}{c}K_{m} \cdot 10^{4} \\
\text { nach (1) }\end{array}$ & $\begin{array}{c}U \\
\text { nach (3) }\end{array}$ \\
\hline 3 & 960 & 3,3 & 1,31 & 13,65 & 0,75 \\
4 & 755 & 4,25 & 1,00 & 13,25 & 0,70 \\
5 & 552 & 5,8 & 0,61 & 11,05 & 0,42 \\
6 & 425 & 7,5 & 0,38 & 8,95 & 0,15 \\
7 & 390 & 6,2 & 0,355 & 9,25 & 0,19 \\
8 & 358 & 8,95 & 0,35 & 9,50 & 0,22 \\
9 & 332 & 9,5 & 0,315 & 9,50 & 0,22 \\
10 & 313 & 10,2 & 0,305 & 9,75 & 0,25 \\
11 & 280 & 11,4 & 0,28 & 10,0 & 0,28 \\
12 & 260 & 12,3 & 0,27 & 10,76 & 0,38 \\
13 & 212 & 15,1 & 0,26 & 12,3 & 0,58 \\
14 & 160 & 20,0 & 0,23 & 14,4 & 0,85 \\
15 & 147 & 21,8 & 0,22 & 14,97 & 0,92 \\
16 & 129 & 27,2 & 0,196 & 15,9 & 0,95 \\
\hline
\end{tabular}

Tab. 3. Bestimmung der Uneinheitlichkeit an einer Reihe hydrolytisch abgebauter Ramie-Cellulosen $\left(P_{0}=3200 ; k_{m}=7,8 \cdot 10^{-4}\right)$

vorgeführten Ramie-Cellulose bestimmt; das Ergebnis gibt Tab. 3 wieder ${ }^{19}$. Man erkennt, daß $U$ bei Präp. $6(\beta=7,5)$ durch ein ausgeprägtes Minimum geht. Es ist dasselbe Präparat, dessen Verteilungskurve in Abb. 1 gezeigt wurde.

Dieses Ergebnis ist neben 3 anderen in Abb. 3 graphisch aufgetragen. Man sieht in allen Fällen ein starkes Minimum in der Gegend des Abbaugrades $\beta=6$. Links vom Minimum, also bei höheren Polymerisationsgraden steigt die Uneinheitlichkeit etwas stärker an, als auf Grund der theoretischen Kurven (Abb. 2) zu erwarten ist. Das liegt wahrscheinlich daran, daß die Anfangsbedingungen des Abbaus schwer exakt zu gestalten sind (es wäre etwa denkbar, daß beim Beginn des Abbaus einzelne Faserpartien schneller angegriffen ẁerden als andere). Interessant ist, daß Baumwoll- und Ramie-Cellulose sich gegenüber hydrolytischen Agenzien ziemlich stark unterscheiden, jedoch beim oxydativen Abbau innerhalb der Fehlergrenzen übereinstimmen.

Aus einem Vergleich der Abbildungen 2 und 3 ergibt sich, daß der Abstand der „Lockerstellen“ etwa 500 Glucosereste beträgt. Genaue Fraktionierungen ergaben, daß die Länge dieser Abschnitte $510 \pm 25$ Glucosereste beträg ${ }^{6}$; eine Cellulosekette vom Polymerisationsgrad 3200 ist dadurch in $6 \mathrm{Ab}$ schnitte unterteilt. Das Verhältnis der Geschwindigkeitskonstanten $k_{a} / k_{b}$ ist beim sauren Abbau für Baumwoll-Cellulose etwa $10^{3}$, für Ramie-Cellulose etwa $10^{4}$; beim oxydativen Abbau beträgt für beide Cellulosearten $k_{a} / k_{b}$ etwa $5 \cdot 10^{5}$.

${ }^{13}$ E. Huss emann, unveröffentlichte Versuche. 
Diese Versuche zeigen, daß das Cellulosemolekül nicht so eintönig gebaut ist, wie man nach den früheren Arbeiten annehmen konnte. Es besitzt eine Architektur, welche sich in den großen Perioden zeigt. Das Ziel weiterer Versuche ist es nun, die chemische Natur der „Lockerstellen“ näher auf-

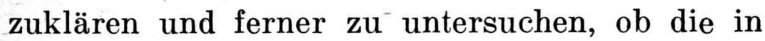
jedem einzelnen Molekül auftretenden Perioden auch im biologischen Zusammenhang, d. h. beim Aufbau der Fasern, eine Rolle spielen.

\section{Reaktionskinetische}

Charakterisierung der schneller spaltenden Bindungen

\section{a) Fragestellung}

Auf Grund der im vorigen Abschnitt besprochenen Versuche sind in einem Cellulosemolekül 0,2\% der Bindungen chemisch von der Hauptmenge der $\beta$-glucosidischen Bindungen verschieden. Der Versuch, einen so geringen Anteil durch chemische Analyse zu charakterisieren, stößt auf außerordentliche Schwierigkeiten, jedoch ist es möglich, hier auf einem indirekten Weg weiterzukommen. Diese Bindungen müßten sich nämlich bemerkbar machen, wenn man die Geschwindigkeit des Abbaus reaktionskinetisch verfolgt, da dieser - grob gesagt - zuerst rasch, dann nach Aufspaltung der „Lockerstellen“ langsamer verlaufen muß. Ermittelt man nun die Geschwindigkeit des Abbaus unter verschiedenen Bedingungen (unter Umständen nach chemischen Eingriffen), so müßte man durch Vergleich mit Modellsubstanzen zu einer chemisch immer engeren Charakterisierung dieser Gruppen kommen können.

Diese Methode ist zuerst von Freuden ber $\mathrm{g}^{20}$ und W. Kuhn ${ }^{21}$ angewandt worden. Diese Forscher bestimmten die Geschwindigkeit, mit welcher die bei der Spaltung neu auftretenden aldehydischen Endgruppen entstanden. Sie konnten so durch systematische Versuche zeigen, daß die Hauptmasse aller Bindungen $\beta$-glucosidischer Natur ist. Dieser Methode ist durch die Analysengenauigkeit eine Grenze gesetzt, so daß wenige Sondergruppen mit abweichender Geschwindigkeitskonstante durch sie nicht mehr erfaßt werden. Ist der Analysenfehler beispielsweise 1\% - tat-

${ }^{20}$ K. Freuden ber g und Mitarb., Ber. dtsch. chem. Ges. 63, 1510 [1930].

${ }^{21} \mathrm{~W} . \mathrm{K} \mathrm{u} \mathrm{h} \mathrm{n}$, ebenda 63, 1503 [1930].

22 A. af Ekensta m m, ebenda 69, 549 [1936]; Ưber Celluloselösungen in Mineralsäuren, Lund 1936. sächlich wird er etwas größer sein -, so werden $1 \%$ schneller spaltende Bindungen nicht mehr erfaßt; das sind aber etwa 30 Bindungsstellen im Gesamtmolekül, die so der Analyse entgehen.

Das Anfangsstadium des Abbaus kann man sehr viel besser verfolgen, indem man die Abbaugeschwindigkeit an Hand der Abnahme des Polymerisationsgrades mißt, wie es als erster Eken$\mathrm{sta} \mathrm{m} \mathrm{m}^{22}$ getan hat. Dieser Forscher bestimmte die Abbaugeschwindigkeit in Phosphorsäure, worin sich Cellulose homogen löst. Nach einigen Verbesserungen der Ekenstammschen Methode konnten G.V.Schulz und Hj.Löhmann ${ }^{23}$ auf diese Weise nachweisen, daß ungefähr 99,8\% aller Bindungen gleichartig, also $\beta$-glucosidischer Natur sind. Es bleiben hiernach noch ungefähr 5-10 Bindungen unbestimmt, also gerade diejenigen Bindungen, welche auf Grund der im vorigen Abschnitt beschriebenen Versuche einen abweichenden Charakter haben müssen.

Durch eine weitere Verfeinerung der Versuchstechnik gelang es dann dem einen von uns gemeinsam mit G. Sing ${ }^{24}$, eine vollständige reaktionskinetische Analyse des Celluloseabbaus durchzuführen, und zwar zunächst für den oxydativen $\mathrm{Ab}$ bau. Wir wollen auf die Methode etwas näher eingehen.

\section{b) Experimentelle und rechnerische Grundlagen}

Gereinigte Baumwollcellulose wurde unter strengstem Sauerstoffausschluß in Schweizers Reagens aufgelöst. Der Polymerisationsgrad bleibt hierbei erhalten. Durch diese Lösung wird dann ein kräftiger und konstanter, mit $\mathrm{NH}_{3}$ gesättigter Luft- oder Sauerstoffstrom hindurchgeleitet (bei konstanter Temperatur), wobei ein allmählicher Abbau eintritt. Nach bestimmten Zeiten wird eine Probe aus dem Reaktionsgefäß herauspipettiert und in CuCl-haltige Schweizer-Lösung eingegossen, wodurch die Reaktion sofort unterbrochen wird. Der Polymerisationsgrad dieser Probe wird bestimmt. Die Messung geschieht viscosimetrisch, nachdem man sich vorher durch vergleichende viscosimetrische und osmotische Messungen die Kenntnis der Beziehung zwischen Viscositätszahl und mittlerem Polymerisationsgrad in dem gesamten durchmessenen Bereich verschafft hat.

${ }^{23}$ G. V.Schulz u. Hj. Löhmann, J.prakt. Chem. 157, 238 [1941].

${ }_{24}$ G. V.Schulz u. G. Sing, unveröffentlichte Versuche. 
Um aus dem zeitlichen Gang des Polymerisationsgrades die Geschwindigkeitskonstante des Abbaus zu berechnen, muß man eine Annahme über die Reaktionsordnung machen. Da es sich um eine Reaktion zwischen Cellulose und Sauerstoff handelt,wobei die Sauerstoffkonzentration während der ganzen Reaktionszeit konstant gehalten wird, nimmt man im allgemeinen an, daß die Reaktion nach der ersten Ordnung verlaufen müßte. Wir werden jedoch sehen, daß auch die nullte Ordnung auftreten kann. Ist letzteres der Fall, so ist die Geschwindigkeitskonstante durch die Gleichung

$$
k_{o}=\frac{c_{g}}{t}\left(\frac{1}{\overline{P_{t}}}-\frac{1}{\bar{P}_{0}}\right)
$$

zu berechnen. Hierbei bedeutet $c_{g}$ die Cellulosekonzentration in Mol Glucoseeinheiten pro $l, P_{o}$ den Anfangspolymerisationsgrad und $\bar{P}_{t}$ den mittleren Polymerisationsgrad zur Zeit $t$.

Verläuft die Reaktion nach der ersten Ordnung, so ist die Geschwindigkeitskonstante

$$
k_{I}=\frac{1}{t} \ln \frac{1-1 / P_{0}}{1-1 / \bar{P}_{t}} .
$$

Entwickelt man den Logarithmus in eine Reihe und vernachlässigt die höheren Glieder, so erhält $\operatorname{man}$

$$
k_{I}=\frac{1}{t}\left(\frac{1}{\bar{P}_{t}}-\frac{1}{P_{0}}\right) \text {. }
$$

Diese Gleichung wurde bereits von $\mathrm{Ek}$ e $\mathrm{n}$ s t a $\mathrm{m} \mathrm{m}^{22}$ benutzt. Über ihre Genauigkeit vergl. G. V.S ch u l z und $\mathrm{Hj}$. Lö hm an n ${ }^{23}$.

Die Gleichungen (5) und (6) kommen folgendermaßen zustande. Es seien im Liter $n$ Mole spaltbarer Bindungen enthalten. Werden diese nach einem Geschwindigkeitsgesetz der nullten Ordnung gespalten, so gilt

$$
d n / d t=-k_{0},
$$

wobei $k_{0}$ von den Reaktionsbedingungen (Temperatur, Konzentration der Kupferionen, des Sauerstoffs, Ammoniaks usw.) abhängt. - Verläuft die Reaktion nach der ersten Ordnung, so ist

$$
d n / d t=-k_{I} n,
$$

wobei für $k_{I}$ das gleiche gilt wie für $k_{0}$.

Allgemein gilt

$$
\frac{d \bar{P}}{d t}=\frac{d \bar{P}}{d n} \frac{d n}{d t} .
$$

Da jedes Cellulosemolekül einen Glucoserest mehr als glucosidische Bindungen enthält, sind im Liter $c_{g}-n$ Mole makromolekularer Substanz. Andrerseits ist dieselbe Molzahl gleich $c_{g} / \bar{P}$. Aus beidem ergibt sich

$$
c_{g}-n=c_{g} / \bar{P} \text {, }
$$

und weiter

$$
d \bar{P} / d n=\overline{P^{2}} / c_{g} .
$$

Setzt man nun (11) in (7) und (9) ein, so erhält man Gl. (5). Setzt man dagegen (11) in (8) und (9) ein, s.s erhält man (6) bzw. (6a).

Sind nur Bindungen einer Art im Cellulosemolekül vorhanden, so muß $k_{0}$ oder $k_{I}$ während des ganzen Abbauvorganges konstant sein. Schneller spaltende Bindungen wirken sich so aus, daß die Konstante während der Reaktion abfällt. Es sei noch bemerkt, daß man durch Geschwindigkeitsmessungen zwar Art und Zahl der reaktionsfähigen Gruppen ermitteln kann, jedoch keinen Aufschluß über deren Anordnung in der Kette erhält. Letzteres erfährt man durch die im Abschnitt I beschriebenen Versuche.

\section{c) Ergebnisse der reaktionskinetischen Analyse}

Wir geben nachfolgend nur einen Teil aus einem größeren Versuchsmaterial wieder, welches wir baldmöglichst an anderer Stelle ausführlich publizieren wollen. Hier soll nur das Grundsätzliche der Methode und der mit ihr erhaltenen Ergebnisse gebracht werden.

In Tab. 4 ist der Reaktionsverlauf des Sauerstoffabbaus für 4 Konzentrationen dargestellt, und die Geschwindigkeitskonstanten $k_{0}$ und $k_{I}$ nach den Gleichungen (5) und (6) berechnet. Wie zu erwarten, sind die für die „Konstanten“ erhaltenen Werte zeitabhängig: Der Abbau verläuft zu Beginn der Reaktion schneller und erreicht erst nach längerer Reaktionsdauer einen Grenzwert der Geschwindigkeit. Die schneller spaltenden Gruppen zeigen sich also auch im Geschwindigkeitsverlauf.

Eine genauere Betrachtung der gewonnenen Meßreihe zeigt allerdings, daß die Verhältnisse noch etwas komplizierter sind, als auf Grund der im vorigen Abschnitt beschriebenen Versuche erwartet werden konnte (vergl. Abb.4). Zunächst einmal konnte aus den oben angeführten Gründen erwartet werden, daß die Reaktion mit Hilfe einer Geschwindigkeitsgleichung der ersten Ordnung beschrieben werden kann. Dann müßte $k_{I}$ unabhängig von der Cellulosekonzentration sein. Das ist offensichtlich nicht der Fall: Je höher $c$ ist, um so stärker fällt $k_{I}$ ab. Ferner zeigen die Kurven eine kleine Ausbuchtung nach mittleren Reaktionszeiten.

Der ersterwähnte Befund läßt sich dadurch erklären, daß die beiden Arten von Bindungen nach verschiedenen Geschwindigkeitsgesetzen spalten, wie man aus folgendem erkennt: Betrachten wir 


\begin{tabular}{|c|c|c|c|}
\hline $\begin{array}{l}\text { Zeit } \\
\text { min. }\end{array}$ & $\bar{P}$ & $\begin{array}{c}k_{o} \cdot 10^{7} \\
\text { nach }(5)\end{array}$ & $\begin{array}{c}k_{I} \cdot 10^{5} \\
\operatorname{nach}(6)\end{array}$ \\
\hline \multicolumn{4}{|c|}{$c_{g}=0,62 \cdot 10^{-2}(1,0 \mathrm{~g} / \mathrm{l})$} \\
\hline 0 & 3200 & - & - \\
\hline 4,5 & 1060 & 8,9 & 14,4 \\
\hline 9 & 700 & 7,8 & 12,6 \\
\hline 18 & 405 & 7,5 & 12,1 \\
\hline $27, \check{0}$ & 245 & 8,5 & 13,8 \\
\hline 47 & 160 & 7,85 & 12,7 \\
\hline \multicolumn{4}{|c|}{$c_{g}=1,24 \cdot 10^{-2}(2,0 \mathrm{~g} / \mathrm{l})$} \\
\hline 0 & 3200 & - & - \\
\hline 4,5 & 1170 & 15,1 & 12,2 \\
\hline 9 & 770 & 13,7 & 11,1 \\
\hline 18 & 480 & 12,2 & 9,9 \\
\hline 47 & 189 & 13,1 & 10,3 \\
\hline 96 & 116 & 10,8 & 8,7 \\
\hline \multicolumn{4}{|c|}{$c_{g}=2,45 \cdot 10^{-2}(4,0 g / l)$} \\
\hline 0 & 3200 & - & - \\
\hline 4,5 & 1070 & 34,8 & 14,1 \\
\hline 9 & 845 & 24,2 & 9,8 \\
\hline 18 & 602 & 18,7 & 7,6 \\
\hline 47 & 335 & 14,1 & 5,8 \\
\hline 145 & 139 & 11,7 & 4,73 \\
\hline 596 & 61 & 6,7 & 2,7 \\
\hline \multicolumn{4}{|c|}{$c_{g}=3,7 \cdot 10^{-2}(6,0 g / l)$} \\
\hline 0 & 3200 & - & - \\
\hline 4,5 & 1060 & 50,5 & 14,4 \\
\hline 9 & 925 & 31,0 & 8,4 \\
\hline 28 & 640 & 16,3 & 4,4 \\
\hline 97,5 & 340 & 10,0 & 2,7 \\
\hline 247 & 166 & 8,6 & 2,3 \\
\hline 596 & 100 & 6,0 & 1,5 \\
\hline
\end{tabular}

Tab. 4. Zeitlicher Verlauf des oxydativen Celluloseabbaus (Ausgangsmaterial Baumwoll-Cellulose vom Polymerisationsgrad 3200; Abbau mit Sauerstoff bei $0^{\circ} \mathrm{C}$ ).

die Anfangswerte in Tab. 4, so sehen wir, daß diese in 4 Meßreihen für $k_{I}$ ziemlich gut übereinstimmen, während die Anfangswerte von $k_{0}$ sehr stark von der Cellulosekonzentration abhängen. Betrachten wir jedoch die Endwerte, so stimmen diese wieder für $k_{0}$ sehr viel besser als für $k_{I}$ überein. Da nun die Reaktionsgeschwindigkeit am Anfang hauptsächlich von den ,schnellen“ Gruppen, am Ende von den „langsamen“ Gruppen bestimmt wird, müssen wir aus unserem Befund schließen, daß die ,schnellen“ Gruppen nach der ersten und die ,langsamen“ Gruppen nach der nullten Ordnung reagieren.

Der Sachverhalt wird noch deutlicher bei Betrachtung der aus einem größeren Versuchsmaterial in Tab. 5 zusammengestellten Werte. Man sieht, daß unter ganz verschiedenen Bedingungen
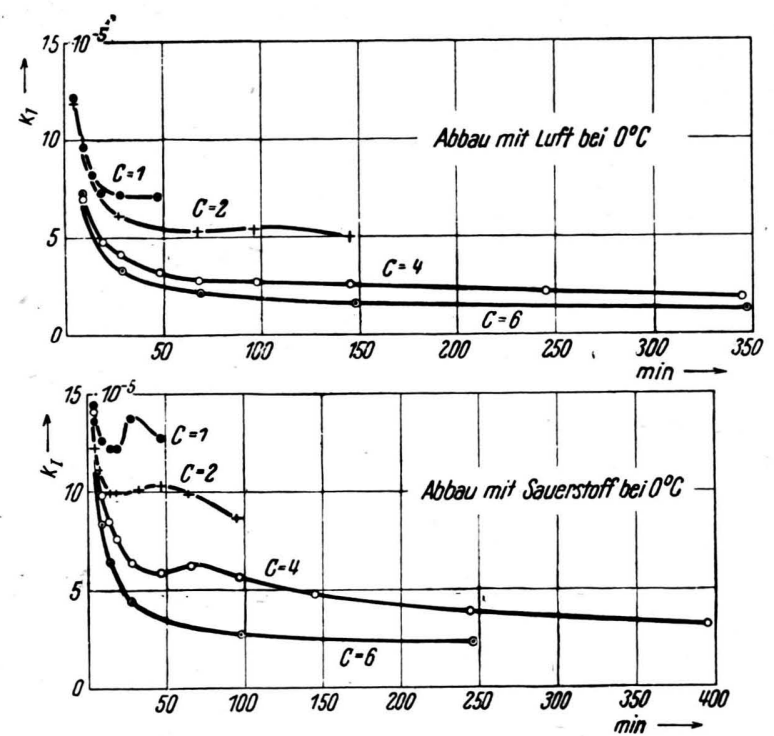

Abb. 4. Zeitlicher Gang der nach der ersten Ordnung berechneten Geschwindigkeitskonstanten (Konzentration $c$ in $g / l$ ).

immer $k_{I}$ für die Anfangswerte und $k_{0}$ für die Endwerte gut übereinstimmt. Also die ,,schnellen“ und die ,langsamen" Bindungen unterscheiden sich nicht nur in der Geschwindigkeit, sondern auch im Mechanismus, nach welchem sie gespalten werden. Indem wir die Versuchsbedingungen noch nach verschiedenen Richtungen variierten und genauere Geschwindigkeitsgleichungen aufstellten, konnten wir die beiden Arten von Bindungen in folgender Weise reaktionskinetisch charakterisieren:

1. Die Hauptmasse der Bindungen ( $\beta$-glucosidisch) spaltet in bezug auf die Cellulosekonzentra-

\begin{tabular}{|c|c|c|c|c|}
\hline \multirow{2}{*}{$c_{g} \cdot 10^{2}$} & \multicolumn{2}{|c|}{$k_{o} \cdot 10^{7}$} & \multicolumn{2}{|c|}{$k_{I} \cdot 10^{5}$} \\
\hline & Ánfang & Ende & Anfang & Ende \\
\hline \multicolumn{5}{|c|}{ Abbau mit Luft bei $0^{n} \mathrm{C}$} \\
\hline 0,62 & 7,5 & $4,4)$ & 9,7 & 7,2 \\
\hline 1,24 & 14,8 & 6,4 & 10,0 & 5,2 \\
\hline 2,45 & 17,4 & 4,8 & 7,0 & 1,9 \\
\hline 3,70 & 27,5 & 4,0 & 7,2 & 1,2 \\
\hline \multicolumn{5}{|c|}{ Abbau mit Sauerstoff bei $0^{\circ} \mathrm{C}$} \\
\hline 0,62 & 8,9 & 8,2 & 12,6 & 13,2 \\
\hline 1,24 & 15,1 & 11,5 & $12,2 l_{131}$ & 9,3 \\
\hline 2,45 & 34,8 & 7,2 & $14,13^{13,1}$ & 2,9 \\
\hline 3,70 & 50,5 & 7,8 & $13,6^{\prime}$ & 1,9 \\
\hline \multicolumn{5}{|c|}{ Abbau mit Luft bei $20^{\circ} \mathrm{C}$} \\
\hline $\begin{array}{l}1,24 \\
3,70\end{array}$ & $\begin{array}{l}19,4 \\
64,5\end{array}$ & $\left.\begin{array}{l}27,6 \\
26,7\end{array}\right\} 27,2$ & $\left.\begin{array}{l}17,7 \\
17,4\end{array}\right\} 17,6$ & $\begin{array}{r}22,3 \\
7,1\end{array}$ \\
\hline
\end{tabular}

Tab. 5. Anfangs- und Endwerte der Geschwindigkeitskonstanten bei Variation der Cellulosekonzentration. 
tion nach der nullten Ordnung. Die Spaltung ist proportional der Wurzel aus der SauerstoffionenKonzentration und unabhängig von der Kupferionen-Konzentration. Sie hat eine Aktivierungsenergie von 19,6 kcal/Mol.

2. In geringer $\mathrm{Zahl}$ vorhandene Bindungen spalten nach der 1. Ordnung mit einer wesentlich höheren Geschwindigkeit. Auch bei diesen ist die Geschwindigkeitskonstante der Wurzel aus der Sauerstoffkonzentration proportional und unabhängig von der Kupferionen-Konzentration. Ihre Aktivierungsenergie beträgt $8,6 \mathrm{kcal} / \mathrm{Mol}$.

Formal lassen sich diese Verhältnisse dadurch erklären, daß die Cellulose nicht direkt mit dem Sauerstoff reagiert, sondern daß hierbei ein Katalysator wirksam ist (wahrscheinlich Hydroxylionen). Bei den ,schnelleren“ Bindungen bildet der Katalysator mit dem einen Reaktionspartner einen Arrheniusschen, im anderen Falle einen van t'H of f schen Zwischenkörper. - Wegen der verschiedenen Reaktionsordnung der beiden Bindungen hängt ihr Geschwindigkeitsverhältnis übrigens von der Cellulosekonzentration ab.

Die Ausbuchtung in den Geschwindigkeitskurven schließlich kann man dadurch erklären, daß eine zweite Art schnell spaltender Gruppen in der Cellulose enthalten ist, welche nach einem 2-stufigen Mechanismus reagieren, wobei der Zerfall der Kette erst in oder unmittelbar nach der 2. Reaktionsstufe eintritt.

Es ist hier nicht der Platz, um das ganze Material, das wir für die Begründung unserer Auffassung besitzen, auszubreiten; das soll baldmöglichst an anderer Stelle geschehen. Jedoch wollen wir noch kurz auf die Frage nach der chemischen Natur der verschiedenen Gruppen eingehen. Nach einer Arbeit von Staudinger und R o o ${ }^{25}$ geht der oxydative Abbau in 3 faßbaren Oxydationsstufen vor sich, wobei der Übergang zur ersten - langsam, von dieser zur zweiten schneller und schließlich der zur dritten noch schneller geht. Es liegt nahe, anzunehmen, daß die beiden schneller spaltenden Gruppen die erste und die zweite Oxydationsstufe darstellen. Hierdurch wäre der einund der zweistufige Mechanismus der beiden schneller spaltenden Gruppen gut verständlich.

25 E. Roos, Dissertat. Freiburg 1941.

26 Wir nahmen früher an (vergl. Zitat 6), daß Carboxylgruppen oder Xylosegruppen für die raschere Spaltung verantwortlich sind. Gemeinsame Versuche mit K. Maurer u. Drehfahl zeigten indessen, daß für die oxydativ spaltenden Gruppen diese Annahme nicht zutrifft.
Für die $\beta$-glucosidischen Bindungen dagegen ist der sehr viel langsamere Vorgang, der zur ersten Oxydationsstufe führt, geschwindigkeitsbestimmend. Wie St a u dinger und $\mathrm{R}$ o os zeigten, kann man durch Einwirkung bestimmter Reagenzien die anoxydierten Gruppen blockieren und damit ihre Spaltung verhindern. Indem man solche Eingriffe vornimmt und anschließend Geschwindigkeitsmessungen macht, kann man schließlich zu einer indirekten, aber recht sicheren chemischen Charakterisierung der verschiedenen Gruppen kommen ${ }^{26}$. Wir hoffen, bald in dieser Richtung weitere Ergebnisse zu erhalten.

\section{Die Lagerung der Lockerstellen im Faserverband}

Falls die pflanzlichen Fasern analog den in der Einleitung erwähnten tierischen Fasern aufgebaut sind, ist zu erwarten, daß die "Lockerstellen“ in senkrecht zur Faserachse liegenden Ebenen angeordnet sind. Diese müßten Abstände von etwa $2500 \AA$ haben, entsprechend 500 Glucoseresten zu je $5 \AA$. Für einen röntgenographischen Nachweis sind diese Abstände noch zu groß, jedoch können sie, wie wir nachfolgend zeigen, bei geeigneter Präparationsmethode mit dem Elektronenmikroskop sichtbar gemacht werden.

Um in den submikroskopischen Aufbau pflanzlicher Fasern einzudringen, hat man sie schon mehrfach nach einer mechanischen Vorbehandlung im Elektronenmikroskop untersucht ${ }^{27}$. Im allgemeinen fanden jedoch dabei starke Deformierungen statt. Mahlt man jedoch Fasern naß in einer Kugelmühle, so werden sie in feine, glatte Fibrillen aufgeteilt, wie der eine von uns mit A. Ca r n ap zeigte $^{28}$. Ein Beispiel hierfür zeigt Abb. 5 A. Man ist versucht, nach dem Vorgang von Hess und Wergin ${ }^{29}$ anzunehmen, daß diese Fibrillen vorgebildete Elemente einer Biostruktur seien. Das ist aber nicht der Fall, denn Kunstfasern, die durch starke Streckung orientiert sind, können in fast derselben Weise in derartige Fibrillen zerfallen, wie Abb. 5 B zeigt 28 a. Auf einer der älteren Aufnahmen $^{27}$ erschien bereits eine gewisse periodische Unterteilung der Fibrillen; jedoch konnte dieser Effekt nicht reproduziert werden.

\footnotetext{
${ }^{27}$ K. Heß, H. Kiessig u. J. Gundermann, Z. physik. Chemie. (B) 49, 64 [1941].

${ }_{28}$ E. Husemann, Cellulosechemie 1944, 132, und unveröffentlichte Versuche mit A. Carnap.

28 a E. H u s e m a n n. Cellulosechemie 1944, 132.

${ }^{29}$ W. We r g in, Kolloid-Z. 98, 132 [1942].
} 
Auf Grund der oben beschriebenen Versuchsergebnisse sollte die erwartete periodische Struktur dann zutage treten, wenn man durch vorherige chemische Behandlung der Fasern die Lockerstellen durchtrennt. Falls diese in Ebenen angeordnet sind, müßten die Fibrillen, in welche die Fasern bei mechanischer Behandlung zerfallen, senkrecht durchschnitten werden und somit in kleinere $\mathrm{Ab}$ - außerordentlich feine kleinere Einheiten. Unter dem Übermikroskop erhält man dann Bilder wie die in Abb. $5 \mathrm{D}, \mathrm{E}$ und $\mathrm{F}$ gezeigten. Bemerkenswert ist die außerordentliche Ähnlichkeit so verschiedener Fasern wie Baumwolle, Ramie und Arundo donax. Kunstfasern dagegen zeigen nicht diesen Zerfall. Sie geben zunächst fibrilläre Unterteilungen, die dann bei wachsendem chemischem Abbau

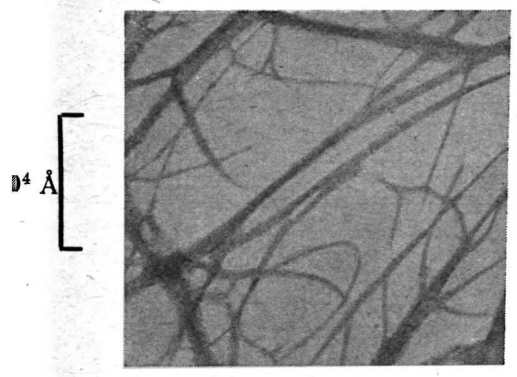

A

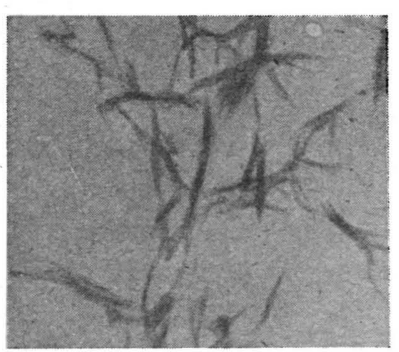

D

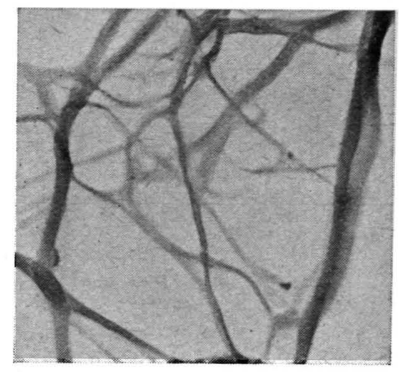

B

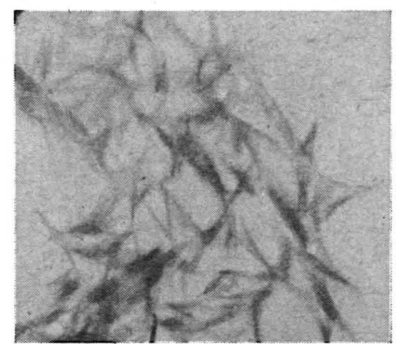

E

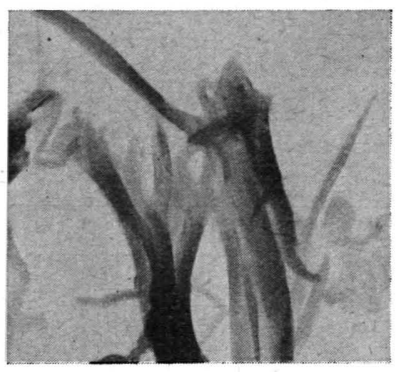

C

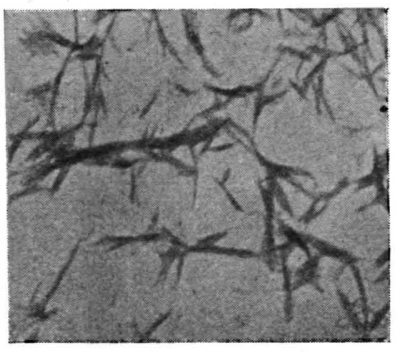

F

Abb. 5. Ubermikroskopische Aufnahmen verschieden vorbehandelter Fasern. Vergr. 1/15000.

A. Baumwolle, naß gemahlen.

B. Gestreckte Acetylseide, verseift (Rodiaseta), naß gemahlen.

C. Dasselbe, mit $\mathrm{HCl}$ abgebaut $(\bar{P}=50)$.

schnitte mit einer der chemischen Periodizität entsprechenden Länge zerfallen. Diese Annahme wurde in folgender Weise nachgeprüft ${ }^{30}$ :

An nativen Fasern wurde durch Behandlung mit Wasserstoffionen ein chemischer Abbau bis zu einem Polymerisationsgrad von etwa 150 bis 50 durchgeführt. Um die Lockerstellen quantitativ aufzuspalten, muß man stärker als bis zum Polymerisationsgrad 500 abbauen; sonst bleibt ein Teil der „schnelleren“ Gruppen ungespalten, da auch immer ein gewisser Bruchteil der ,normalen“ Bindungen durchtrennt wird. Derart chemisch vorbehandelte Naturfasern zerfallen bereits bei schwacher Quetschung unter einem Deckgläschen in

so E. Husemann und A. Carnap, J. makromol. Chem. 1, 16 [1943].
D. Baumwolle, hydrolytisch abgebaut $(\bar{P}=80)$ und gequetscht.

E. Ramie, ebenso behandelt $(\bar{P}=85)$.

F. Arundo donax, ebenso behandelt $(\bar{P}=150)$.

unscharfe Ränder bekommen und schließlich verschmieren*.

Quetscht man die Präparate etwas stärker, so zerfallen die in Abb. $5 \mathrm{D}$ bis $\mathrm{F}$ gezeigten spindelartigen Körperchen in noch feinere stäbchenförmige Partikel, die besonders dann gut gesehen werden, wenn man die Substanz stark verdünnt. Leider lassen sich diese wegen ihrer Feinheit sehr schlecht reproduzieren, so daß wir in den $\mathrm{Ab}$ bildungen nur die an konzentrierterem Material erhaltenen Aufnahmen wiedergeben. Immerhin sind in Abb. $5 \mathrm{~F}$ solche elementaren Einheiten zu erkennen.

* Dieses Verhalten zeigen nur Fasern, die im Fällungsbad gestreckt sind. Vergl. A. Si p pel, Z. Elektrochem. 50 [1944]. 
Die Länge dieser Einheiten wurde an einem gröBeren Material genauer vermessen. Hierzu wurden von jedem Faserpräparat Aufnahmen bei höherer Verdünnung in größerer Anzahl gemacht, und dann die Länge jeder erkennbaren, frei liegenden Partikel gemessen. Hatte man für ein bestimmtes Präparat etwa 150 derartige Meßwerte, so wurde der prozentuale Anteil der Längen von 500 zu $500 \AA$ ausgerechnet und graphisch aufgetragen. Um bei der Auswertung unabhängig von zufälligen Versuchsbedingungen zu werden, wurde auch der Abbaugrad variiert. Die auf diese Weise erhaltenen Kurven sind in Abb. 6 und 7 dargestellt $^{31}$. Man erkennt in allen Fällen ein scharfes Maximum für Partikellängen zwischen 2000 und $2500 \AA$. Diese Länge ist innerhalb der Fehlergrenze übereinstimmend mit der Länge der chemischen Perioden. Sie ist ferner unabhängig vom Polymerisationsgrad, bis $\mathrm{zu}$ welchem abgebaut wurde, und völlig identisch bei Baumwolle und Ramie.

Die mittlere Länge der Partikel scheint etwas kleiner zu sein (etwa 10\%) als die Länge der chemischen Molekülabschnitte. Das ist aber wahrscheinlich dadurch bedingt, daß sich die Enden der Teilchen nicht völlig scharf abbilden. Eine Reihe ganz verschiedenartiger Einflüsse könnte einen solchen Effekt herbeiführen, z. B. ein etwas bevorzugter Abbau vom Ende her ${ }^{23}$ oder eine Unschärfe der Enden, dadurch hervorgerufen, daß die Ketten sich dort etwas voneinander abspreizen.

Eine weitere Frage ist die, warum die auftretenden Partikel nicht alle gleich groß sind. Daß größere Partikel auftreten, liegt wahrscheinlich daran, daß nicht alle ausgemessenen Teilchen völlig einzeln lagen. Bei ihrer z. Tl. außerordentlichen Feinheit kann es leicht vorkommen, daß gelegentlich ein für einfach gehaltenes Teilchen aus mehreren elementaren Partikeln besteht. Schwerer zu erklären ist das Auftreten kleinerer Partikel. Hier deutet manches darauf hin, daß außer der Hauptperiode noch kürzere Perioden existieren. Vielleicht ist hierauf das schwache-Maximum bei der halben Länge der Hauptperiode zurückzuführen. Einige weitere Versuche legen die Vermutung nahe, daß die hydrolytisch spaltenden Sondergruppen von den oxydativ spaltenden verschieden sind und auch in verschiedenen Netzebenen liegen. Versuche, die hieriiber in Gang waren, sind leider durch die Zerstörung des Laboratoriums und des Utbermikroskops unterbrochen worden.

Die übermikroskopisch gefundenen Faserabschnitte sind nicht identisch mit den röntgenographisch aufgefundenen Mizellen. Diese haben eine Länge von im Mittel $700 \AA$, sind also ungefähr 3mal kleiner als unsere Teilchen ${ }^{32}$. Die Dicke der Teil79.

31 E. Husemann u. A. Carnap, Naturwiss. 1944, 32 Vergl. O. K r atk y, Kolloid. Z. 96, 301 [1940].

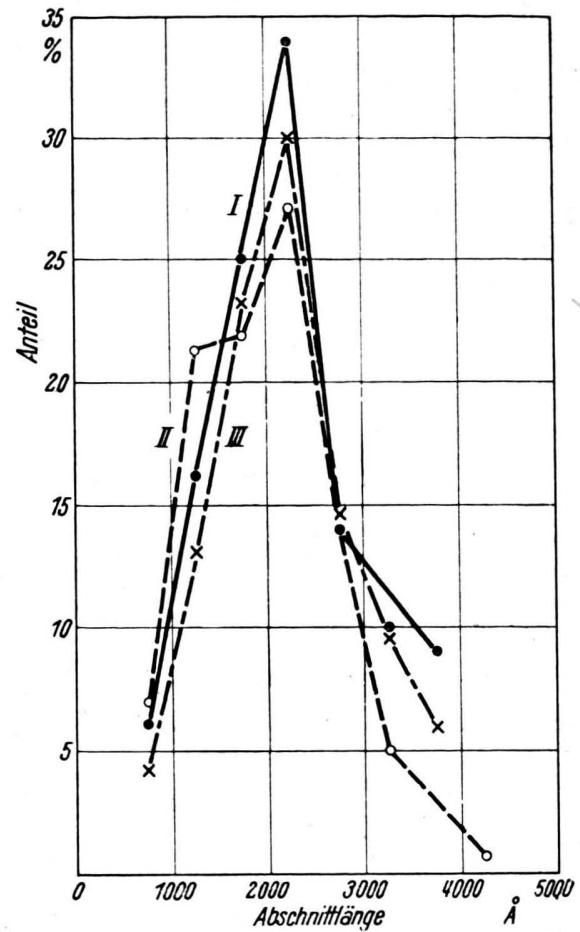

Abb. 6. Häufigkeitsverteilung der Partikellängen von hydrolytisch abgebauten Baumwollfasern.
I : $\overline{\mathrm{P}} 150$
II : $\overline{\mathrm{P}} 70$
III: $\overline{\mathrm{P}} 90$

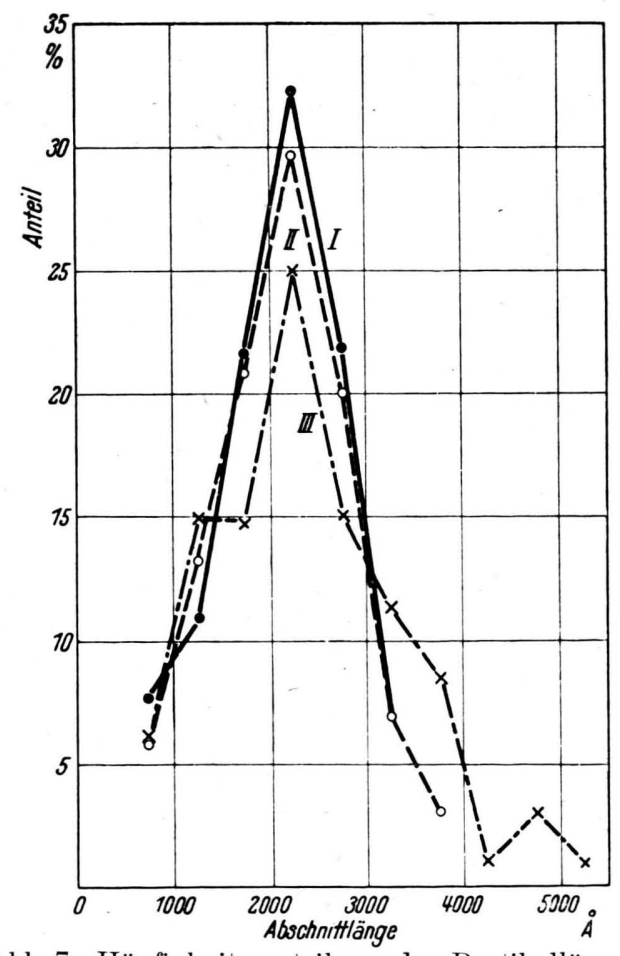

Abb. 7. Häufigkeitsverteilung der Partikellängen von hydrolytisch abgebauten Ramiefasern.
I : $\overline{\mathrm{P}} 180$
II : $\overline{\mathrm{P}} 130$
III : $\overline{\mathrm{P}} 80$ 
chen wurde maximal zu etwa $300 \AA$ gefunden. Nach unten $\mathrm{zu}$ ist sie nicht begrenzt; die feinsten Teilchen liegen hart an der Auflösungsgrenze, d. h. sie haben Durchmesser von etwa $50 \AA$. Man kann ohne weiteres annehmen, daß auch noch feinere Teilchen vorhanden sind, die aber nicht mehr sichtbar gemacht werden können. Die wichtigste Aufgabe weiterer Untersuchungen ist es nun, die Lage der von uns aufgefundenen Elementarteilchen in der Faser aufzuklären.

Die hier beschriebene Forschungsrichtung setzt es sich zum Ziel, in möglichst vollständiger Weise den Zusammenhang der mikroskopischen Strukturen mit dem chemischen Aufbau der Organismen aufzuklären. Methodisch ist das Bindeglied zwischen diesen beiden Größenbereichen die Röntgenmethode und die Übermikroskopie. Heute erscheint es nicht mehr als ausgeschlossen, daß man durch Entwickelung entsprechender Methoden den submikroskopischen Bereich so weit in die Hand bekommt, daß man ein Bild der Organismen gewinnen kann, welches die biologischen Strukturen bis in die feinsten Einzelheiten, nämlich bis in die Lagerung der Moleküle und Atome hinein, zu überblicken erlaubt.

IV. Zur Entstehung der makromolekularen Stoffe im Organismus

Versucht man, sich eine Vorstellung darüber zu machen, in welcher Weise die Cellulose in der Pflanze entsteht, so stößt man auf folgende Schwierigkeit. Nimmt man etwa an, daß die Pflanze in einem gleichmäßig fortlaufenden Prozeß $500 \mathrm{Glu-}$ cosereste aneinanderreiht, um dann regelmäßig eine andere Bindung einzuschieben, so müßte man voraussetzen, daß die Pflanze bis 500 zählen kann. Da dieses sehr unwahrscheinlich ist, steht nur einer der beiden folgenden Wege offen:

1. Die Abschnitte bestehen ziemlich genau aus $2^{9}=512$ Glucoseresten. Man könnte sich nun eine gekoppelte Reihe von Fermenten denken, welche suczessiv Ketten von 2, 4, 8, 16 usw. bis 512 Glucoseeinheiten aufbauen. Bei der bekannten Spezifität der Fermente wäre jeder derartige Schritt als ein chemisch besonderer Vorgang aufzufassen.

2. Der andere Weg wäre der, daß die Kettenlänge der Moleküle und ihr periodischer Aufbau durch

${ }^{33}$ H. St a u d ing e r u. R. Si gn er, Lieb. Ann. Chem. 474, 267 [1929]. vorgegebene biologische Strukturen gesteuert werden.Bei synthetischenOperationen im Laboratorium ist das Reaktionsgefäß stets groß gegen die Moleküldimensionen; daher ist die Größe von Makromolekülen, die wir durch Polymerisation oder Kondensation herstellen, immer nur durch einen statistischen Mittelwert gegeben, um welchen die wirklichen Molekülgrößen in einem weiten Bereich streuen. In den Organismen jedoch sind submikroskopische Strukturen in der Größe der Makromoleküle bereits vorgebildet, die unter geeigneten Umständen deren Bildungsprozesse bis in die Einzelheiten hinein lenken könnten.

Nachdem wir jetzt erfahren haben, daß sich die Struktur der Makromoleküle in übermolekulare Zellstrukturen fortsetzt, gewinnt die zweite Möglichkeit sehr an Wahrscheinlichkeit. Schon früher hatten Sta udinger und Sig ne ${ }^{33}$ vermutet, daß die Cellulose nicht im Protoplasma gebildet wird, um dann ín den Fasern abgelagert zu werden, sondern daß die Kondensation der Glucosemoleküle zur Cellulose an der Oberfläche der Fasern vor sich geht, so daß die Cellulose bereits während ihrer Entstehung in das Gitter eingeordnet ist.

Hiernach erscheint die biologische Struktur als das Primäre gegenüber dem chemischen Vorgang ${ }^{34}$. Um bei unserem obigen Bild zu bleiben: In der Biochemie ist das Reaktionsgefä $ß, d . h$. im weitesten Sinne das Milieu, in welchem sich die Reaktionen abspielen, diejenige Seite der Vorgänge, die das eigentlich Biologische und damit auch Biochemische ausmacht. Diese Auffassung erscheint als besonders naturgemäß, wenn man es als wichtigstes Kennzeichen lebender Organismen ansieht, daß diese in ihrer charakteristischen Gestalt fremde Substanz assimilieren und dadurch wachsen und sich vermehren.

Das Ziel der bisher in der Biochemie vorwiegend ausgeübten Forschungsrichtung ist es, die Lebensvorgänge als eine Summe chemischer Reaktionen aufzufassen, welche sich - wenigstens prinzipiell - auch getrennt vom Organismus in vitro abspielen können. Jetzt scheint es aber, als ob die mit dem Wachstum zusammenhängenden chemischen Reaktionen, also gerade die für den Lebensprozeß fundamentalen, an das ordnende Milieu des leben-

${ }^{34}$ Diese Auffassung wird heute wohl am schärfsten von Frey-Wyssling vertreten, dessen Formulierung ,,structura omnis e structura“ hierfür charakteristisch ist (vergl. etwa ,Submikroskopische Struktur des Protoplasmas und seiner Derivate“, B ornträg e r, Berlin 1938, S. 287). 
den Organismus mit seiner vorgegebenen Feinstruktur gebunden sind. Indessen wird hierdurch die Bedeutung der ersterwähnten Forschungsrichtung nicht vermindert; indem man sie mit äußerster Konsequenz weiterverfolgt, wird man um so genauer erkennen, an welcher Stelle ihr etwa eine Grenze gesetzt ist.

Allerdings ist nach allen bisherigen Erfahrungen nicht anzunehmen, daß sich eine derartige Grenze in der Durchbrechung irgendwelcher grundlegender physikalisch-chemischer Gesetze (etwa der Hauptsätze der Thermodynamik und ihrer Folgerungen) äußert. Nirgends scheint das Leben diesen Gesetzen zu widersprechen, aber es erscheint von ihnen aus gesehen als etwas äußerst Unwahrscheinliches. Ein Symptom hierfür ist die praktisch unendliche Mannigfaltigkeit möglicher Molekülformen auf makromolekularem Gebiet ${ }^{35}$ und demgegenüber die Beschränkung dieser Möglichkeiten bei den biologisch wichtigen Stoffen im Sinne bestimmter Normen, die sich z. B. in der Svedbergschen Regel über die Molekulargewichte der Proteine, den periodischen Strukturen der tierischen und pflanzlichen Faserstoffe und auch in anderen biologischen "Gesetzen“" zeigen ${ }^{36}$. Schon das Auftreten einheitlicher makromolekularer Stoffe ist in diesem Sinne etwas Unwahrscheinliches.

An dieser Stelle erhebt sich die Frage: Sind diese Regeln Folgerungen rein physikalisch-chemischer Gesetzmäßigkeiten (etwa durch geometrische Be-

35 Vergl. G. V.Schulz, J. makromol. Chem. 1, 36 [1943]. dingungen oder solche der Stabilität von Makromolekülen oder überhaupt makromolekularer Systeme hervorgerufen), oder handelt es sich bei ihnen um Ausflüsse spezifisch biologischer Notwendigkeiten? Im letzten Fall wären sie, ohne mit physikalisch-chemischen Gesetzen in Widerspruch zu stehen, doch nicht aus ihnen zu erklären. Sie wären dann als ein System von Bedingungen aufzufassen, die den speziellen Ablauf chemisch-physikalischer Vorgänge in ganz bestimmte Bahnen lenken, während ihre Herkunft als etwas ,historisch" Gegebenes anzusehen ist. Nach dieser Herkunft zu fragen, wäre erst dann sinngemäß, wenn man das Wechselspiel der Bedingungen, ihren inneren Zusammenhang und schließlich ihre Zusammenfassung in der Einheit des lebenden Organismus übersieht.

Wir sind noch weit entfernt von einer bündigen Antwort auf diese Fragen. Aber eine wichtige Voraussetzung dafür, sie in präziser Weise stellen und beantworten zu können, wird zweifellos dadurch geschaffen, daß man den Aufbau der Organismen bis hinab zu den molekularen und atomaren Strukturen erforscht und so - zunächst rein beschreibend - ein vollständig zusammenhängendes Bild von dem chemischen Aufbau über die submikroskopischen Strukturen bis hinauf zu den lebenden Gestalten der Organismen zu gewinnen sucht. Wir hoffen, durch unsere hier beschriebenen Untersuchungen einen Beitrag zur Lösung dieser Fragen liefern zu können.

36 Vergl. L. v. B ertalanf fy, Theoretische Biologie, B or n träg e r, Berlin 1932 und 1941. 